\title{
ARTÍCULO GIENTIFFICO
}

\section{EL ROL DEL OFICIAL DE GUMPLIMIENTO Y LOS PROGRAMAS DE WTECRIDAD EN LAS ORGANZAGIONES}

\section{THE ROLE OF THE COMPLIANCE OFFICER AND INTEGRITY PROGRAMS IN ORGANIZATIONS}

PAULA A.

\section{D'ONOFRIO}

Universidad de Buenos Aires, Ciudad Autónoma de Buenos Aires, Argentina.

$\bowtie$ pdonofrio1@gmail.com

\section{PALABRAS CLAVE}

Compliance, integridad, programas de autoevaluación

\section{KEYWORDS}

Compliance, integrity, self-assessment programs

\section{RESUMEN}

El presente trabajo tiene por fin revisar los resultados de una encuesta realizada en el segundo semestre del año 2019, en el marco del proyecto de Desarrollo Estratégico (PDE) de la Universidad de Buenos Aires PDE_9_2019 "Corrupción, fraude empresario y sistemas de cumplimiento en las organizaciones".

Las preguntas están dirigidas a los participantes internos cualquiera sea el rol que ocupan dentro de la organización. El objetivo de esta encuesta es poder determinar la importancia que las organizaciones les dan a los sistemas de Compliance. Las respuestas consideradas fueron 70 . Si bien los resultados no son categóricos, podemos observar que la figura del Compliance ocupa un posicionamiento jerárquico adecuado, lo que debería darle independencia y recursos dentro de la estructura. Sin embargo, aún queda un camino por recorrer en relación con las capacitaciones y el entrenamiento acerca de elementos claves del programa de integridad, considerados necesarios de acuerdo con los términos de la Ley 27.401.

\section{ABSTRACT}

This article aims at reviewing the results of a survey carried out in the second semester of 2019 as part of the Strategic Development Project (PDE) of the University of Buenos Aires PDE_9_2019 "Corruption, business fraud and compliance systems in organizations".

The questions are addressed to internal participants, regardless of their role within the organization. The purpose of this survey is to determine the importance attached to compliance systems by organizations.

Seventy answers were considered. Although the results are not categorical, we can observe that the Compliance figure holds an adequate hierarchical position, which should provide it with independence and resources within the structure. However, further progress needs to be made in relation to training and education on key elements of the integrity program, considered necessary under the terms of Law No. 27,401.

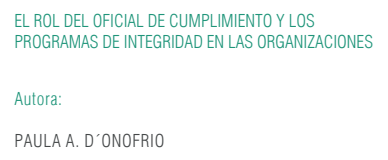




\section{INTRODUCCIÓN}

El presente trabajo se enmarca dentro de proyecto de Desarrollo Estratégico (PDE) ${ }^{1}$ de la Universidad de Buenos Aires PDE_9_2019 "Corrupción, fraude empresario y sistemas de cumplimiento en las organizaciones", bajo mi dirección. Este tiene por fin el desarrollo de herramientas del sistema de información que permitan establecer el correcto funcionamiento de los sistemas de cumplimiento en las organizaciones.

En Argentina, a partir de la publicación de la Ley 27.401 el 1 de diciembre de 2017, reglamentada por el Decreto Reglamentario 277/2018 del 6 de abril de ese año, muchas organizaciones comenzaron a implementar o revisar sus programas de integridad. En el marco de la ley argentina, un programa de integridad es un conjunto de acciones, mecanismos y procedimientos internos que adopta una empresa para promover la integridad en la organización, y que buscan prevenir, detectar y corregir irregularidades y delitos asociados a la corrupción.

Como parte del proyecto, en el segundo semestre del año 2019, se realizó una encuesta a empleados de organizaciones con el fin de identificar, por un lado, la importancia que se le da a la figura del Compliance, de acuerdo con el posicionamiento jerárquico en la estructura, y, por el otro, el nivel de conocimiento que estas personas tienen acerca del funcionamiento de algunos elementos claves de los programas de integridad.

Este trabajo en particular busca analizar los resultados de la encuesta realizada, que no tiene por su número de respuestas la posibilidad de fijar tendencia, pero nos resulta muy orientadora a la hora de detectar algunos riesgos significativos en el funcionamiento de dichos programas de autocontrol de acuerdo con las hipótesis de trabajo.

\section{LOS PROGRAMAS DE INTEGRIDAD DE ACUERDO A LA LEY 27.401}

La propuesta legislativa en relación a la actual Ley 27.401 (2017) obedeció a la necesidad de que la Argentina cumpliera con los requisitos para ingresar como Estado miembro a la Organización para la Cooperación y el Desarrollo Económicos (OCDE), a los compromisos asumidos frente al G20, a las recomendaciones del Grupo de Acción Financiera Internacional (GAFI) y a los Lineamientos de las convenciones internacionales de lucha contra la corrupción, ratificadas e incorporadas a nuestro derecho interno?
La ley promueve la cooperación público-privada para incentivar la autoregulación empresarial, mediante las ventajas que acompañan a los programas de integridad descriptos en su artículo 23.

Aunque no establece en sus términos la obligatoriedad de presentarlos, en su artículo 24 lo menciona como requisito para ser proveedor del Estado nacional. Del mismo modo, antes del proceso penal aquellas organizaciones que presenten estos mecanismos de autorregulación, si se autodenunciasen y devolvieran el beneficio indebido, se permite la eximición de la pena y responsabilidad administrativa (Ley 27.401, 2017, art. 9); por su parte, el artículo 8 lo considera un atenuante en la graduación de la sanción penal una vez fijada la sentencia; y el artículo 18 lo define como una condición necesaria para acceder a un acuerdo de colaboración eficaz durante el proceso.

La norma determina que el programa deberá contener, al menos, los siguientes elementos (Ley 27.401, 2017, art. 23):

Un código de ética o de conducta, o la existencia de políticas y procedimientos de integridad aplicables a todos los directores, administradores y empleados, independientemente del cargo 0 función ejercidos, que guíen la planificación y ejecución de sus tareas para prevenir la comisión de los delitos bajo la ley 27.401.

- Reglas y procedimientos específicos para prevenir ilícitos en el ámbito de concursos y procesos licitatorios, en la ejecución de contratos administrativos 0 en cualquier otra interacción con el sector público.

Capacitaciones periódicas sobre el programa de compliance a directores, administradores y empleados.

Adicionalmente, la ley establece que el programa podrá contener Ios siguientes elementos:

Análisis periódico de riesgos y la consecuente adaptación del programa de integridad.

- Apoyo visible e inequívoco al programa de integridad por parte de la alta dirección y gerencia.

Canales internos de denuncia de irregularidades, abiertos a terceros y adecuadamente difundidos.

- Una política de protección de denunciantes contra represalias.

Un sistema de investigación interna que respete los derechos de los investigados e imponga sanciones efectivas a las violaciones del código de ética o conducta.

Procedimientos que comprueben la integridad y trayectoria de terceros o socios de negocios, incluyendo proveedores, distribui-

\footnotetext{
1PDE: Tienen como propósito elaborar instrumentos de promoción científico-tecnológica que revaloricen e incrementen su aporte al desarrollo socio-productivo. Son proyectos interdisciplinarios que cuentan con una entidad que actúa como referente de interés del proyecto.

2Convención de las Naciones Unidas contra el Tráfico llícito de Estupefacientes y Sustancias Psicotrópicas - Ley 24.072. Convención Interamericana contra la corrupción - Ley 24.759. Convención Internacional Contra la Delincuencia Organizada Transnacional y protocolos complementarios - Ley 25.632. Convención Interamericana Contra el Terrorismo - Ley 26.023. Convención de las Naciones Unidas Contra la Corrupción - Ley 26.097. Protocolo contra la Fabricación y el Tráfico llícitos de Armas de Fuego, sus Piezas y Componentes y Municiones, que complementa la Convención de las Naciones Unidas contra la Delincuencia Organizada Transnacional, adoptado por la Asamblea General de la Organización de las Naciones Unidas el 31 de mayo de 2001 - Ley 26.138.
} 
EL ROL DEL OFICIAL DE CUMPLIMIENTO Y LOS PROGRAMAS DE INTEGRIDAD EN LAS ORGANIZACIONES I Paula A. D’Onofrio I Proyecciones Revista digital Instituto de Investigaciones y Estudios Contables - FCE UNLP I No14 (enero - diciembre 2020) 14-21 I D0I https://doi.org/10.24215/26185474e009 dores, prestadores de servicios, agentes e intermediarios, al momento de contratar sus servicios durante la relación comercial. - La debida diligencia durante los procesos de transformación societaria y adquisiciones, para la verificación de irregularidades, de hechos ilícitos o de la existencia de vulnerabilidades en las personas jurídicas involucradas.

- El monitoreo y evaluación continua de la efectividad del programa de integridad.

- Un responsable interno a cargo del desarrollo, coordinación y supervisión del programa de integridad.

- El cumplimiento de las exigencias reglamentarias que sobre estos programas dicten las respectivas autoridades del poder de policía nacional, provincial, municipal o comunal que rija la actividad de la persona jurídica.

Se deja asentado que dicho sistema deberá guardar relación con "Ios riesgos propios de la actividad que la persona jurídica realiza, su dimensión y capacidad económica” (Ley 27.401, 2017, art. 22).

De acuerdo al artículo 1 del Decreto Reglamentario 277/2018, será la Oficina Anticorrupción del Ministerio de Justicia y Derechos Humanos quien establecerá los lineamientos y guías que resulten necesarios para el mejor cumplimiento de lo establecido en los artículos 22 y 23 de la Ley 27.401 (2017).

\section{DEFINICIÓN DEL PROBLEMA Y JUSTIFICACIÓN}

Tomamos como hipótesis de trabajo las siguientes:

Una organización comprometida

1. dota a la figura del Compliance de independencia y posicionamiento jerárquico para desempeñar su tarea;

2. tiene políticas escritas que demuestren sus valores, su compromiso, y comunica, capacita y entrena a todo su personal en la importancia del Compliance.

La figura del Compliance, esté representada por un individuo 0 un departamento, tiene por fin establecer procedimientos que aseguren el cumplimiento normativo interno y externo. Es decir, no solo vela por el cumplimiento de la ley, sino también por los valores organizacionales. Es una función independiente que identifica, asesora, alerta, monitorea y reporta los riesgos de cumplimiento.

Es importante destacar que esta función debe estar dotada por el órgano de administración de la organización de autonomía y recursos materiales, tecnológicos y humanos necesarios para desarrollar su cometido. A tales efectos se le delegarán facultades y competencias suficientes para desarrollar su tarea sin precisar autorización, manteniendo la objetividad, imparcialidad e independencia (D’Onofrio, 2018).

Dentro el organigrama de la organización esta función debe ocupar una posición que le otorgue capacidad para dirigirse de forma autónoma a otras áreas o funciones de la organización y garantice un acceso rápido, así como la comunicación fluida con el órgano de administración social y sus comités delegados. Por otra parte, la retribución de las personas que integran la función de Compliance no podrá depender de los objetivos de negocios ni los resultados económicos de la organización (Asociación Argentina de Etica y Compliance, 2017).

Para poder relevar el nivel de conocimiento de las personas que trabajan en una organización acerca del funcionamiento de los programas de integridad elegimos dentro del cuestionario hacer énfasis en la misión, la visión, el código de conducta, las capacitaciones en relación al tema y la línea de denuncia.

Una organización que posee políticas escritas que demuestren un claro compromiso con las conductas éticas y transparentes, de las cuales se desprende el código de conducta, es un comienzo para considerar interés en temas de integridad. Es un elemento clave para quien genere un programa de integridad que apunte a superar el mero cumplimiento de la ley.

Debemos entender que estas políticas y procedimientos son dinámicos, deben adaptarse a la organización y al contexto donde se desarrollan las actividades concretas, por lo tanto, deben ser revisadas periódicamente.

Sin embargo, la mera existencia de estos valores, aun en forma de código, no implica que toda la organización los conozca y se comprometa. Esto es producto de un aprendizaje continuo inductivo y participativo de los distintos agentes de la organización. De acuerdo con los participantes y la exposición que tengan frente a conductas mencionadas en estos valores y códigos, es que se hace necesario una capacitación continua.

Es de suma importancia establecer los mecanismos para que dicho código sea conocido por todos los partícipes alcanzados en su definición, ya que es una realidad que en muchas organizaciones es casi un elemento declamativo que no es transmitido a los miembros de la organización (D’Onofrio, 2018).

Como señala Regner, es difícil imaginar un entrenamiento en ética (Durrieu y Saccani, 2018, pp. 212-213), pero muchas veces puede resultar útil remarcar conductas positivas mediante una comunicación clara y precisa, incluso mediante juegos e infografías. Cada organización debe ajustar la comunicación a las necesidades de su personal de forma tal que no queden dudas de su comprensión.

Por último, es importante destacar que la mayoría de los delitos de la Ley 27.401 llegan a la luz por medio de denuncias; por ello contar con un canal resulta imprescindible. Estos canales pueden ser anónimos 0 no, pero siempre deben proteger la identidad del denunciante y actuar de manera rápida con protocolos previamente definidos.

Como mecanismos formales de revelaciones anónimas tenemos, por ejemplo, las líneas de denuncias, correos y sitios web que pueden ser utilizados tanto por usuarios internos como externos. Muchas veces un mero buzón dentro de la organización puede ayudar a conocer situaciones que se perciben como contrarias a las políticas organizacionales o a la misma ley. 
EL ROL DEL OFICIAL DE CUMPLIMIENTO Y LOS PROGRAMAS DE INTEGRIDAD EN LAS ORGANIZACIONES I Paula A. D’Onofrio I Proyecciones Revista digital Instituto de Investigaciones y Estudios Contables - FCE UNLP I No14 (enero - diciembre 2020) 14-21 I D0I https://doi.org/10.24215/26185474e009
Lo más importante es que quienes sean sus usuarios tengan confianza en el correcto funcionamiento. En algunas organizaciones estos canales se administran externamente, dando, tal vez, un mayor viso de seguridad; sin embargo, estamos convencidos de que el éxito de estos canales dependerá de la claridad con que se transmita su utilidad, de las garantías que se brinden y, fundamentalmente, de las acciones que se tomen una vez recibida la denuncia.

\section{LA ENCUESTA}

Las preguntas están dirigidas a los participantes internos cualquiera sea el rol que ocupan dentro de la organización.

El objetivo de esta encuesta es poder determinar la importancia que las organizaciones les dan a los sistemas de Compliance. Como primera medida la posición jerárquica que ocupa la figura del Compliance dentro de la organización será clave para detectar su grado de independencia y posicionamiento dentro de la estructura. Luego las preguntas se orientan a conocer el funcionamiento de elementos claves dentro de los sistemas de Compliance a través de los empleados de la organización. En este punto centramos el análisis en la misión, la visión, el código de conducta y la línea de denuncia 0 de transparencia, estos deberían ser transmitidos al personal de manera clara mediante el proceso de inducción y las capacitaciones periódicas que también son motivo de la encuesta.

Metodológicamente, esta encuesta tiene algunas limitaciones, ya que, si bien se hizo una difusión amplia a través de los integrantes del proyecto y de grupos de trabajo a los que estos pertenecen, la cantidad de respuestas obtenidas fue de 74 pero se debió eliminar 4 por inconsistencias. La investigación aseguraba la confidencialidad de las respuestas.

Como corolario, el sector al que pertenecen los encuestados nos permitirá comparar y/o profundizar análisis posteriores.

El relevamiento se realizó durante el segundo semestre de 2019.

Las preguntas fueron:

1- ¿Cuál es su rol dentro de la organización?

Un dato interesante es que las 4 encuestas que debieron eliminarse por presentar contradicciones fueron respondidas por empleados, 10 que denota que no tenían un claro conocimiento sobre el Compliance. La composición de la muestra terminó siendo la siguiente:

Tabla № 1: Rol de los encuestados

\begin{tabular}{|r|r|r|r|r|r|r|}
\hline & & & & $\begin{array}{c}\text { FORMA } \\
\text { PARTE DEL } \\
\text { DPTO. DE } \\
\text { DIRECTIVO }\end{array}$ & $\begin{array}{l}\text { GERENTION } \\
\text { CIESGOS } \\
\text { COMPLIANCE }\end{array}$ & Y SOX \\
\hline 8 & 15 & 10 & 26 & 10 & 1 & TOTAL \\
\hline
\end{tabular}

Fuente: Elaboración propia en base a relevamiento
Creemos que la variedad es enriquecedora ya que un $33 \%$ responde a líneas jerárquicas y un 14\% forma parte de área de Compliance. Por lo que casi la mitad de la muestra debería tener información de primera mano en materia de Compliance si realmente hay un compromiso del Tone from the Top, más allá de lo que planteamos como hipótesis, que todas las personas deben estar informadas con el mismo nivel de conocimiento para asegurar el éxito.

2- La figura del Compliance dentro de la organización está representada por:
a. Una persona de forma individual
b. Un departamento
c. Un equipo

Figura № 1: Representación de la figura del Compliance en la organización

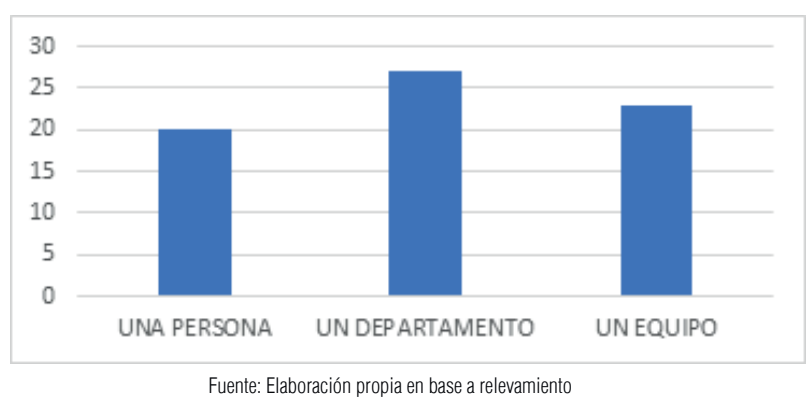

Nos parece llamativo que una buena parte de los encuestados $(28,5 \%)$ marquen a una sola persona como referente del Compliance en la organización.

Esto nos dispara muchos interrogantes, aquí alguno de ellos:

¿Qué características personales debería reunir un individuo para llevar adelante un programa de integridad? ¿Cuáles habilidades blandas debe poseer?

¿Qué herramientas asociadas debería manejar para tener un buen desempeño?

¿Cómo es posible que haga las capacitaciones? ¿Se hacen? ¿Cómo asegura el control?

Claramente esto nos marca una debilidad en cuanto a recursos asignados y un factor a considerar en la matriz de riesgo.

Con relación a las otras respuestas, la idea de que esté representado por un departamento marca la institucionalización y la representatividad dentro de la organización, mientras que la representación por parte de un equipo nos daría la idea de varias personas en la organización que pueden tener diferentes posiciones y que se alinean bajo el liderazgo del Oficial de Cumplimiento. Esto último lo hemos visto como una forma de transición en los últimos tiempos.

3- Dentro del organigrama la figura del Compliance depende (dependencia jerárquica) de:

Respuesta a completar 


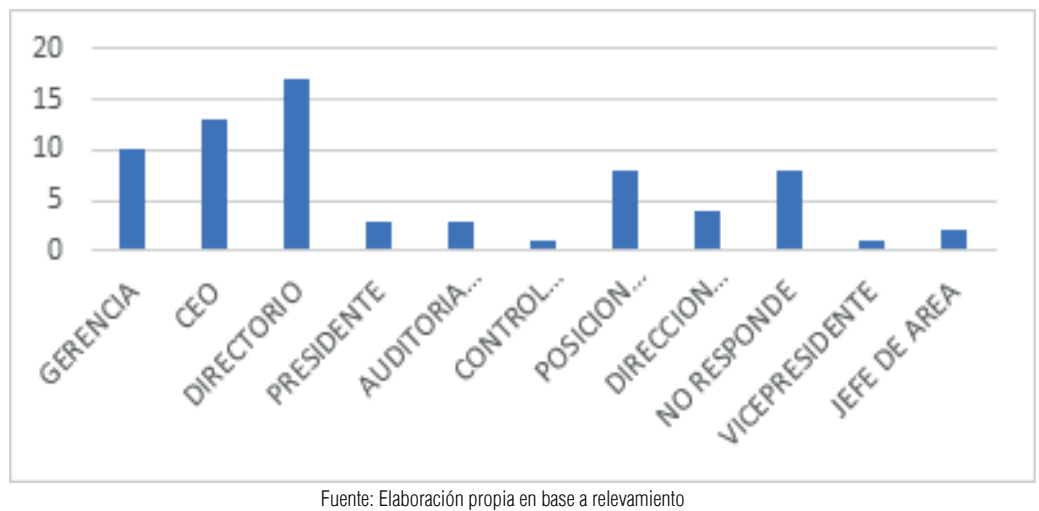

Tabla № 2: Dependencia jerárquica del Compliance datos numéricos

\begin{tabular}{|c|c|c|c|c|c|c|c|c|c|c|}
\hline GERENCAA & CEO & DARECTOSSO & PAESIDENTE & AUDITORIA INTEENA & CONTROL INTEBNO & $\begin{array}{c}\text { POSIOON } \\
\text { IERA AQUUCA NO } \\
\text { ESPEOFICADA }\end{array}$ & $\begin{array}{l}\text { DIRECOON } \\
\text { LEGALES }\end{array}$ & NO RESPONDE & VICEPRESIDENTE & JEFE DE AFEA \\
\hline 10 & 13 & 17 & & 3 & 1 & 8 & & 8 & & \\
\hline
\end{tabular}

Fuente: Elaboración propia en base a relevamiento

Esta respuesta es una de las que nos dio más trabajo interpretar. Como hacíamos referencia al inicio, tal vez porque muchos empleados desconocen la estructura jerárquica de la organización. Por otro lado, enciende también muchas alertas en cuanto a la independencia, representatividad y libertad del Oficial de Cumplimiento.

Si el $11 \%$ desconoce la respuesta, es posible que no les genere confianza y credibilidad esta función, nos preguntamos si irían a denunciar alguna situación si realmente no tienen en claro de quién depende esta figura, entendemos que se desvirtúa la función. Aclaramos que todos los que manifestaron desconocer se autocalificaron como empleados.

Claramente la única respuesta posible es que el Oficial de Cumplimiento dependa de los niveles jerárquicos más altos de la organización y que, en caso de recibir una denuncia que involucre a este, tenga una posibilidad de reporte superior. La independencia en sus funciones tiene que estar acompañada por un rango jerárquico acorde. Una posición de jefatura no permitiría tener peso suficiente en la organización, por más que contara con el apoyo del Directorio; afortunadamente, son muy pocos los que respondieron relacionándolo con este nivel.

Muchas nuevas posiciones de Compliance se relacionan de manera directa con las áreas de Control Interno o Auditoria Interna. El área de Control Interno suele estar más relacionada con el área contable y, si bien creemos que la profesión de contador tiene las habilidades naturales para posiciones de Oficial de Cumplimiento, el rol del control interno es otro. Por su parte, la Auditoria Interna es reconocida como "Tercera línea de defensa", evalúa Ios acontecimientos una vez que ya se produjeron, no es su función evitarlos sino detectarlos. Por tal motivo entendemos que esto podría aceptarse solamente en una etapa de transición.
4- ¿Cómo se aseguran que el personal conozca la visión y misión, y el código de conducta de la organización?:

a. Se lo da para leer al ingresar. 13

b. Firma al ingresar. 14

c. Se le informa que está colgado en la intranet. 9

d. Está dentro del proceso de inducción. 33

e. No responde. 1

Figura № 3: Formas de inducción de la visión y misión, y el código de conducta

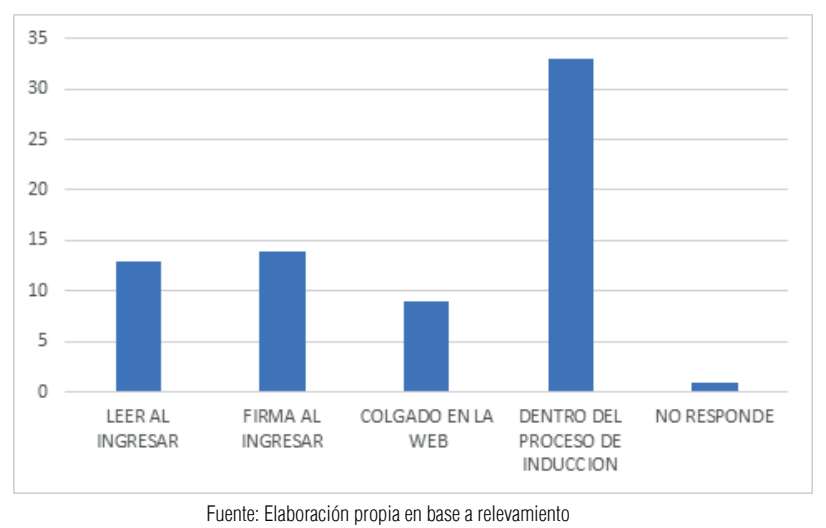

Esta pregunta nos parece clave, la mayoría de las organizaciones cuentan hoy con espacios para difundir la misión y la visión; sin embargo, muchas veces notamos que su personal no se encuentra claramente alineado a ellas, o que ciertas políticas institucionales no concuerdan con lo manifestado en ellas. En general, nos parece satisfactoria la respuesta ya que la mejor manera es dentro del proceso de inducción, pero para que esto realmente refleje un compromiso debe estar ligado a las capacitaciones periódicas, lo cual se relaciona con la pregunta siguiente. 


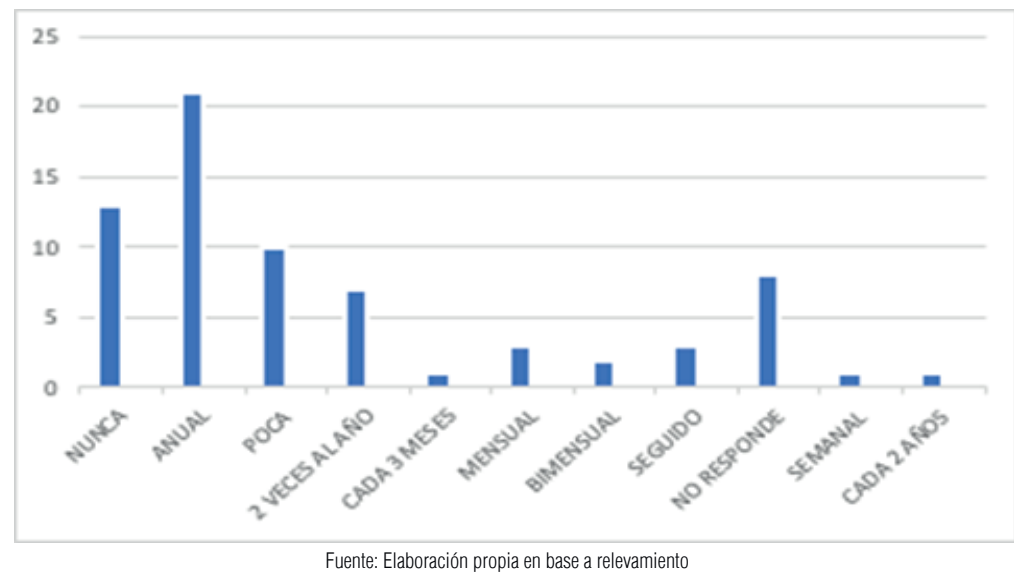

Tabla № 3: Cantidad de capacitaciones en términos numéricos

\begin{tabular}{|c|c|c|c|c|c|c|c|c|c|c|}
\hline NUNCA & ANUAL & POCA & $\begin{array}{l}2 \text { VECES AL } \\
\text { ANNO }\end{array}$ & CADA 3 MES & MENSUAL & BIMENSUAL & SEGUIDO & NO RESPONI & ISEMANAL & CADA 2 AÑOS \\
\hline 13 & 21 & 10 & 7 & 1 & 3 & 2 & 3 & 8 & 1 & 1 \\
\hline & & & & & & & & & & \\
\hline
\end{tabular}

Fuente: Elaboración propia en base a relevamiento

5- ¿Con qué regularidad se hacen capacitaciones en el tema de Compliance y ética organizacional?:

En este caso la respuesta era abierta ya que no queríamos limitar ninguna posibilidad, cada uno debía responder de acuerdo con la organización. Lamentablemente, vemos que el 18\% no capacita, 0 Io hace de manera que el personal reconoce como escasa (14\%), y el $30 \%$ lo realiza anualmente. Creemos que esto es clave en el compromiso de la dirección y en cómo esto se transmite a toda la línea dentro de las organizaciones. La falta de entrenamiento representa un riesgo clave. Si no se percibe dentro de los líderes el claro involucramiento en los valores de una organización, es difícil que en las líneas inferiores pueda derramarse. Por otra parte, Ios entornos cambiantes requieren la actualización de los procedimientos y esto conlleva a realizar nuevas capacitaciones.

Algo que nos parece relevante destacar es que quienes no responden la pregunta en este caso se autodefinen en diferentes posiciones jerárquicas. Hay una persona que se posiciona dentro del departamento; dos, en el nivel gerencial; una, en mandos medios y cuatro que se autodefinen como empleados. Esto denota un riesgo significativo especialmente en aquellas personas comprometidas con la posición.

6- ¿Tiene línea de transparencia?:

Sí

No

El 76\% (53 respuestas afirmativas) indicó que tenían línea de transparencia. Esto es muy positivo porque indica que tienen la posibilidad de denunciar cualquier ilícito o situación de vulnerabi- lidad en la que pudieran encontrarse. Recordemos que el 26\% de Ios que respondieron la encuesta se definieron como empleados, las líneas de transparencia les dan una campana de seguridad.

Figura № 5: Existencia de líneas de transparencia 0 denuncia

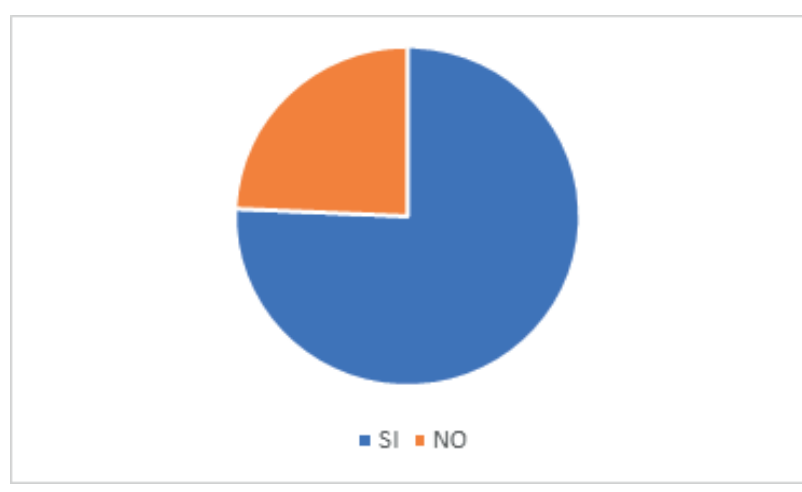

Fuente: Elaboración propia en base a relevamiento

7- Si la respuesta es sí:

Es para uso interno

Es de uso externo

Es de uso interno y externo

Figura № 5: Existencia de líneas de transparencia 0 denuncia

\begin{tabular}{|r|r|l|}
\hline & & INTERNO Y \\
\hline INTERNO & EXTERNO & EXTERNO \\
\hline 19 & 1 & 33 \\
\hline
\end{tabular}

Fuente: Elaboración propia en base a relevamiento 
EL ROL DEL OFICIAL DE CUMPLIMIENTO Y LOS PROGRAMAS DE INTEGRIDAD EN LAS ORGANIZACIONES I Paula A. D'Onofrio I Proyecciones Revista digital Instituto de Investigaciones y Estudios Contables - FCE UNLP I No14 (enero - diciembre 2020) 14-21 I D0I https://doi.org/10.24215/26185474e009
La distribución de las 53 respuestas afirmativas sobre la línea de transparencia se da de la manera indicada en la Tabla № 4, la mayoría presenta canales de denuncia internos y externos, lo que redunda en una muy buena práctica de prevención.

8- ¿Quién administra la línea de transparencia?

Internamente

Externamente

Tabla № 5: Administración de la línea de transparencia

\begin{tabular}{|c|c|}
\hline INTERNA & EXTERNA \\
\hline 42 & 11 \\
\hline
\end{tabular}

Fuente: Elaboración propia en base a relevamiento

Esta pregunta, si bien es más técnica, es lo que daría seguridad sobre la transparencia de la línea en su administración.

Por último, podemos ver las actividades que desarrollan las empresas en donde trabajan los encuestados.

Tabla № 6: Sector autopercibido por los encuestados al que pertenecen las organizaciones

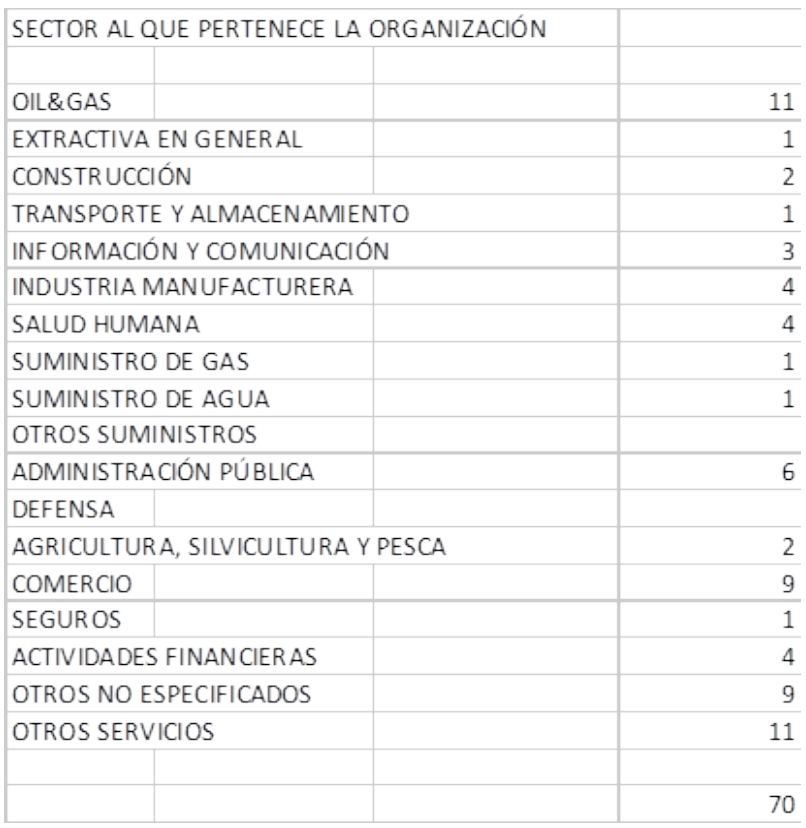

Fuente: Elaboración propia en base a relevamiento

Esta pregunta, si bien es más técnica, es lo que daría seguridad sobre la transparencia de la línea en su administración.

\section{CONCLUSIONES}

Como primer punto entendemos que la ley 27.401 ha dado un claro impulso a la definición de los programas de integridad y ha motiva- do a las organizaciones a revisar sus valores, políticas y procedimientos, del mismo modo que ha permitido la actualización de sus códigos y ha aumentado el compromiso en la comunicación.

En relación con la primera hipótesis planteada respecto de si una organización comprometida dota a la figura del Compliance de independencia y posicionamiento jerárquico para desempeñar su tarea, en base a esta muestra pareciera que sí, ya que, para la mayoría (54\%), dicha figura depende de los altos mandos de la organización y, para un 26\%, de niveles gerenciales en general. Desde el punto de vista estructural, se estaría cumpliendo con una estructura jerárquica adecuada. Adicionalmente, observamos que la mayoría tiene un departamento o un equipo de trabajo, esto daría idea de asignación de recursos, aunque no podemos ser contundentes en esta revelación.

Con respecto a la segunda hipótesis acerca de la existencia de políticas escritas que demuestren su compromiso, y la comunicación, capacitación y entrenamiento a todo su personal en la importancia del Compliance, encontramos diferentes resultados. La mayoría conocía la existencia de los valores organizacionales al incorporarse a la organización; sin embargo, al consultar sobre las capacitaciones y entrenamientos, los resultados fueron poco alentadores; tampoco, según entendemos, existen actualizaciones, y, si existen, no se comunican, ya que la frecuencia es realmente baja.

Por último, la mayoría de los encuestados conocen la existencia de la línea de transparencia y su funcionamiento. Creemos que, si el $76 \%$ de los encuestados manifiestan tener y conocer el funcionamiento de la línea de transparencia, es altamente alentador para evitar situaciones de corrupción, en donde, de acuerdo a los estudios internacionales (Asociación Argentina de Etica y Compliance, 2017), es la manera más común de detectar estos delitos.

Si bien la encuesta no define tendencias porque la muestra ha sido escasa, vemos que las organizaciones han comprendido las ventajas de tener un representante de Compliance. En principio, de acuerdo con la composición y estructura, podríamos interpretar que la figura goza de independencia y recursos, pero aún falta mucho en cuanto a capacitación transversal sobre elementos básicos del programa de integridad.

\section{REFERENCIAS BIBLIOGRÁFICAS}

Asociación Argentina de Ética y Compliance. (2017). Libro Blanco sobre la función de Compliance.

D’Onofrio, P. (2018). La figura de los sistemas de cumplimiento en la nueva ley de responsabilidad penal de las personas jurídicas. Enfoques, (9), 95-109.

Decreto Nacional 277/2018. Boletín Oficial de la República Argentina, 6 de abril de 2018. http://www.saij.gob.ar/277-nacional-reglamentacion-ley-27401-dn20180000277-2018-04-05/123456789-0abc-772- 
0000-8102soterced?utm_source=newsletter-semanal\&utm_medium=email\&utm_term=semanal\&utm_campaign=decreto-nacional.

Durrieu, N. y Saccani, R. R. (Dir.). (2018). Compliance, Anticorrupción y Responsabilidad Penal de las personas jurídicas. La Ley. https://www.thomsonreuters.com.ar/content/dam/openweb/documents/pdf/arg/white-paper/supl_compliance_y_resp_penal_emp _15_mayo.pdf

Ley 24.072. Convención de las Naciones Unidas contra el Tráfico llícito de Estupefacientes y Sustancias Psicotrópicas. Boletín Oficial de la República Argentina, 14 de abril de 1992. http://servicios.infoleg.gob.ar/infolegInternet/anexos/0-4999/471/norma.htm

Ley 24.759. Convención Interamericana contra la corrupción. Boletín Oficial de la República Argentina, 17 de enero de 1997.

http://servicios.infoleg.gob.ar/infoleglnternet/anexos/40000-44999/41466/norma.htm

Ley 25.632. Convención Internacional Contra la Delincuencia Organizada Transnacional y protocolos complementarios. Boletín Oficial de la República Argentina, 30 de agosto de 2002. http://servicios.infoleg.gob.ar/infoleglnternet/verNorma.do?id=77329

Ley 26.023. Convención Interamericana Contra el Terrorismo. Boletín Oficial de la República Argentina, 15 de abril de 2005. http://servicios.infoleg.gob.ar/infolegInternet/anexos/105000-109999/105500/norma.htm

Ley 26.097. Convención de las Naciones Unidas Contra la Corrupción. Boletín Oficial de la República Argentina, 6 de junio de 2006. http://servicios.infoleg.gob.ar/infoleg Internet/anexos/115000-119999/116954/norma.htm

Ley 26.138. Protocolo contra la Fabricación y el Tráfico llícitos de Armas de Fuego, sus Piezas y Componentes y Municiones, que complementa la Convención de las Naciones Unidas contra la Delincuencia Organizada Transnacional, adoptado por la Asamblea General de la Organización de las Naciones Unidas el 31 de mayo de 2001. Boletín Oficial de la República Argentina, 4 de septiembre http://servicios.infoleg.gob.ar/infoleglnternet/anexos/115000-119999/119448/norma.htm

Ley 27.401. Responsabilidad Penal. Boletín Oficial de la República Argentina, 1 de diciembre de 2017. http://servicios.infoleg.gob.ar/infolegInternet/anexos/295000-299999/296846/norma.htm

Saccani, R. y Morales Oliver, G. (2018). Tratado de Compliance. La Ley. 